\section{Immunological Cross-reactivity between Plasma Membrane and Endoplasmic Reticulum of Ehrlich Ascites Carcinoma}

Microsomal membranes of Ehrlich ascites carcinoma can be separated into membrane classes of high and low density, which on the basis of enzyme, physiochemical and immunological criteria ${ }^{1-4}$ are considered to be derived from endoplasmic reticulum and plasma membrane, respectively. Because the membranes from the endoplasmic reticulum lack the antigens involved in the agglutination and/or immune lysis of intact Ehrlich ascites carcinoma by rabbit and horse antisera directed against microsomal membranes of Ehrlich ascites carcinoma ${ }^{1,2}$, we wished to test, by means of antibody-binding, whether' there are not some antigens which the two membrane classes have in common.

Plasma membrane, endoplasmic reticulum and immune horse immunoglobulin were prepared as in earlier work ${ }^{\mathbf{1}, 2}$. The immunoglobulin was labelled with ${ }^{14} \mathrm{C}$-acetic anhy. dride $^{5}$ without loss of agglutinating activity from the carcinoma. Antibody absorption by plasma membrane and endoplasmic reticulum was measured by equilibrating stated amounts of membrane and immunoglobulin in $1 \mathrm{ml}$. of 0.15 molar sodium chloride, 0.005 molar calcium chloride, 0.01 molar tris hydrochloric acid $(p \mathrm{H} 7.4)$ for $16 \mathrm{~h}$ at $0^{\circ} \mathrm{C}$, and then for $0.5 \mathrm{~h}$ at $37^{\circ} \mathrm{C}$. The samples were then made tc $5.0 \mathrm{ml}$. with the buffer and centrifuged for $45 \mathrm{~min}$ at 50,000 r.p.m. and $4^{\circ} \mathrm{C}$ (Spinco $L-2$ ultracentrifuge, rotor $S W 50)$. The supernatants were decanted, the tubes fully drained and the pellets resuspended in $5.0 \mathrm{ml} .0 .15$ molar sodium chloride and 0.005 molar calcium chloride. After another centrifugation at 50,000 r.p.m. and $4^{\circ} \mathrm{C}$ for $45 \mathrm{~min}$, the supernatants wereagaindecanted, the tubes drained and carefully blotted on the inside and the pellets suspended in $0.5 \mathrm{ml}$. 0.15 molar sodium chloride. Samples $(0.05 \mathrm{ml}$.) of these suspensions were mixed with $1.0 \mathrm{ml}$. of hydroxide of hyamine $10 x$ (Packard Instrument Co.) and $10 \mathrm{ml}$. of Bray's solution and counted to 5 per cent error in a Tricarb liquid scintillation counter. The supernatants and appropriate dilutions of unabsorbed immunoglobulin were counted in the same way. All counts were carried out in duplicate as was each absorption experiment. We used two different membrane preparations and two lots of labelled antibody prepared from the same antiserum. To measure antigens common to endoplasmic reticulum and plasma membrane the immunoglobulin was first absorbed with varying amounts of endoplasmic reticulum as described. The pellets of endoplasmic reticulum were then removed by centrifugation and samples of the supernatants equilibrated with a constant amount of plasma membrane for $16 \mathrm{~h}$ at $0^{\circ} \mathrm{C}$ and $30 \mathrm{~min}$ at $37^{\circ} \mathrm{C}$. The plasma membrane pallets were then sedimented, washed and counted. Preliminary studies showed negligible absorption of normal horse immunoglobulin and showed that the washing procedure used was adequate to reduce adventitious counts to insignificant levels. Labelled immunoglobulin was used as the internal standard and results are thus expressed as $\mu \mathrm{g}$ of immunoglobulin absorbed by plasma membrane or endoplasmic reticulum.

Absorption tests with plasma membrane show that, below an immunoglobulin : membrane weight ratio of 2 , the amount of antibody absorbed is constant (about 0.04 $\mathrm{mg} / \mathrm{mg}$ ). Specific absorption declines with higher proportions of antigen and is about $0.02 \mathrm{mg} / \mathrm{mg}$ at a $1: 1$ ratio. With endoplasmic reticulum (Fig. 1, curve a) specific antibody absorption progressively diminishes as the proportion of antigen increases. Maximum antibody binding is attained at an immunoglobulin : endoplasmic reticulum ratio of $0 \cdot 5$. Further increases in the proportion of endoplasmic reticulum do not lead to additional absorption of antibody. When a given amount of antibody $(0.7 \mathrm{mg})$ is first equilibrated with increasing amounts of endoplasmic reticulum (as in Fig. $1 a$ ), and, after removal

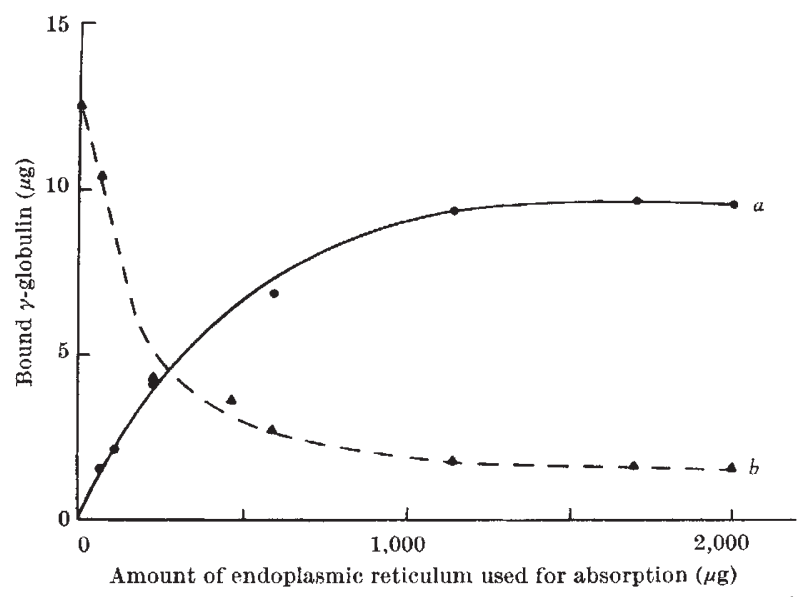

Fig. 1. Antibody-binding by plasma membrane and endoplasmic reticulum, (a) - Antibody-absorption curve of endoplasmic reticulum. $0.7 \mathrm{mg}$ of immunoglobulin was equilibrated with the amounts of endoplasmic reticulum shown, under the conditions given in the text. (b) $\mathbf{A}$ - - A . Decrease in the antibody bound by $0.5 \mathrm{mg}$ of plasma membrane after prior absorption of the immunoglobulin with increasing amounts of endoplasmic reticulum.

of the endoplasmic reticulum, is absorbed with a constant amount $(0.5 \mathrm{mg})$ of plasma membrane, curve $b$ in Fig. 1 results. This shows a reduction in the amount of antibody bound to plasma membrane from $0.025 \mathrm{mg} / \mathrm{mg}$ of membrane in the controls to $0.003-0.004 \mathrm{mg} / \mathrm{mg}$ after absorption with excess endoplasmic reticulum.

These results, which show that there are antigenic sites on plasma membrane which are either lacking or inaccessible in endoplasmic reticulum, are consistent with the previous demonstration ${ }^{1,2}$ of the lack of absorption by endoplasmic reticulum of horse anti-Ehrlich ascites carcinoma agglutinating antibody. On the other hand, the data also imply that the two membrane classes, although differing in a number of critical features, have several structural components in common.

This work was supported by the US Public Health Service.

Donald F. Hoelzl Wallach

Vlasta Vlahovic

Biochemical Research Laboratory,

Massachusetts General Hospital, and

Department of Biological Chemistry,

Harvard Medical School,

Boston, Massachusetts.

Received August 18, 1967.

1 Kamat, V. B., and Wallach, D. F. H., Science, 148, 1343 (1965)

2 Wallach, D. F. H and Kamat, V. B., Methods in Enzymology (edit. by Neufeld, E. F., and (xinsburg, v.), 8, 164 (1966).

${ }^{3}$ Wallach, D. F. H., Kamat, V. B., and Gail, M. L., J. Cell Biol., 30, 601 (1966).

${ }^{4}$ Wallach, D. F. H., and Kamat, V. B., J. Cell Biol., 30, 660 (1966).

${ }^{5}$ Kabat, E. A., Fxperimental Immunochemistry, seeond ed., 803 (Charles 0. Thomas, Springfield, Ill., 1961),

\section{Effects of Phytohaemagglutinin on Homograft Rejection}

Altноugh phytohaemagglutinin (PHA) has been widely investigated in vitro, relatively little is known about its effects in vivo ${ }^{1}$. It has been used in humans with aplastic anaemia, who subsequently showed evidence of increased bone marrow activity in some cases ${ }^{2}$. Gamble ${ }^{3}$ found that intravenous injection of PHA in mice resulted in increases in the cellularity, the proportion of immature cells, and the weight of the spleen, which were most marked 3 days after administration. Calne et al. ${ }^{4}$ used combined PHA and azathioprine ('Imuran') therapy in an attempt to prolong the survival times of renal homotransplants in dogs. Intravenous PHA potentiated the immunosuppres- 